\section{Eliminating Spiked Bovine Spongiform Encephalopathy Agent Activity from Heparin}

\author{
Cyrus Bett, Omozusi Andrews, David M. Asher, \\ Teresa Pilant, David Keire, Luisa Gregori
}

Author affiliations: US Food and Drug Administration, Silver Spring, Maryland, USA (C. Bett, O. Andrews, D.M. Asher, T. Pilant, L. Gregori); US Food and Drug Administration, St. Louis, Missouri, USA (D. Keire)

\section{DOI: https://doi.org/10.3201/eid2610.200142}

US manufacturers, concerned about bovine spongiform encephalopathy (BSE), ceased marketing bovine heparin in the 1990s. Recent short supplies of safe porcine heparin suggest that reintroducing bovine heparin might benefit public health. We purified heparin from crude bovine extract spiked with BSE agent, removing substantial infectivity and abnormal prion proteins (PrP ${ }^{T S E}$ ).

$\mathrm{H}$ eparin is a widely used injectable anticoagulant. In the 1990s, bovine-derived heparin was withdrawn from the US market because of concerns about possible contamination of bovine tissues with the agent of bovine spongiform encephalopathy (BSE), the causative agent of variant Creutzfeldt-Jakob disease (vCJD) in humans (1). Currently, only porcine heparin, mostly from China, is marketed in the United States. The US Food and Drug Administration has encouraged reintroduction of bovine-sourced heparin into the US market to improve the reliability of the heparin supply chain by diversifying sources $(2,3)$.
The risk that BSE agent might contaminate bovine tissues is now very small because of safeguards implemented during the BSE crisis (4).

We previously showed that a model 4-step bench-scale heparin manufacturing process cleared substantial amounts of spiked scrapie agent, a surrogate for BSE agent (5). Our protocol yielded heparin with physicochemical identity, purity, and potency similar to those of United States Pharmacopeia (USP) standard heparin. In this study, we spiked commercial crude bovine heparin with BSE agent itself and processed samples using the same manufacturing process we applied to scrapie agent. We tested each intermediate product for residual abnormal prion protein (PrPTSE, a biochemical marker of BSE) and infectivity. We assayed BSE infectivity using intracerebral inoculations of $30-\mu \mathrm{L}$ volumes into BSE-susceptible transgenic mice (TgBo110) overexpressing the bovine prion-protein-encoding (PRNP) gene (6). To overcome heparin's acute toxicity when administered intracerebrally into mice, we diluted the samples; $10^{-4}$ was the lowest dilution tolerated.

We ended the study 2 years after inoculations, testing brains of all mice for PrPTSE using the HerdCheck BSE-Scrapie Ag Test (IDEXX Laboratories, https://www.idexx.com) (7), which was previously found to be more sensitive than Western blots (8), to assign final disease status (Table). We detected infectivity in samples up to the diatomaceous-earth (DE) filtration step. We estimated removals by DE filtration conservatively, assuming that a 10 -fold lower dilution, not tested, would have infected all mice. Sodium hydroxide $(\mathrm{NaOH})$ treatment removed 1.7 $\log _{10}$ of BSE infectivity and DE filtration removed $\geq 1.1$

\begin{tabular}{|c|c|c|c|c|c|c|c|c|c|c|c|}
\hline \multirow[b]{2}{*}{ Brain dilutions } & \multicolumn{6}{|c|}{ Samples with heparin } & \multicolumn{5}{|c|}{ Samples after heparin removed } \\
\hline & BSE spike & $\begin{array}{c}\mathrm{NaOH} \\
\text { treatment }\end{array}$ & $\begin{array}{c}\mathrm{DE} \\
\text { filtration }\end{array}$ & $\begin{array}{c}\mathrm{H}_{2} \mathrm{O}_{2} \\
\text { bleaching }\end{array}$ & \multicolumn{2}{|c|}{$\begin{array}{l}\text { Final } \\
\text { product }\end{array}$} & $\begin{array}{l}\text { BSE } \\
\text { spike }\end{array}$ & $\begin{array}{c}\mathrm{NaOH} \\
\text { treatment }\end{array}$ & $\begin{array}{c}\mathrm{DE} \\
\text { filtration }\end{array}$ & $\begin{array}{c}\mathrm{H}_{2} \mathrm{O}_{2} \\
\text { bleaching }\end{array}$ & $\begin{array}{c}\text { Final } \\
\text { product }\end{array}$ \\
\hline $10^{-1}$ & NT & NT & NT & NT & \multicolumn{2}{|c|}{ NT } & NT & NT & NT & NT & $3 / 5$ \\
\hline $10^{-2}$ & NT & NT & NT & NT & \multicolumn{2}{|c|}{ NT } & $5 / 5$ & $5 / 5$ & $2 / 4$ & $2 / 5$ & $0 / 5$ \\
\hline $10^{-3}$ & NT & NT & NT & NT & \multicolumn{2}{|c|}{ NT } & $5 / 5$ & $3 / 4$ & $2 / 4$ & $0 / 5$ & $0 / 5$ \\
\hline $10^{-4}$ & $19 / 19$ & $16 / 16$ & $3 / 19$ & $0 / 19$ & \multicolumn{2}{|c|}{$0 / 20$} & $4 / 4$ & $3 / 5$ & $0 / 5$ & $0 / 5$ & $0 / 5$ \\
\hline $10^{-5}$ & $8 / 8$ & $3 / 10$ & $0 / 10$ & $0 / 10$ & \multicolumn{2}{|c|}{$0 / 10$} & $2 / 4$ & $0 / 5$ & $0 / 5$ & $0 / 5$ & $0 / 5$ \\
\hline $10^{-6}$ & $9 / 9$ & $0 / 10$ & $0 / 10$ & $0 / 10$ & \multicolumn{2}{|c|}{$0 / 10$} & $2 / 5$ & $0 / 5$ & $0 / 5$ & $0 / 5$ & NT \\
\hline \multirow[t]{4}{*}{$10^{-7}$} & $0 / 5$ & NT & NT & NT & \multicolumn{2}{|c|}{ NT } & $0 / 5$ & \multicolumn{2}{|l|}{ NT } & NT & NT \\
\hline & \multicolumn{7}{|c|}{ Animal bioassay } & \multicolumn{4}{|c|}{ RT-QulC } \\
\hline & \multicolumn{3}{|c|}{ Samples with heparin } & \multicolumn{4}{|c|}{ Samples after heparin removed } & \multicolumn{4}{|c|}{ Samples after heparin removed } \\
\hline & \multirow{2}{*}{$\begin{array}{c}\log _{10} I D_{50} / g \\
\text { brain }\end{array}$} & \multicolumn{2}{|c|}{$\log _{10}$ removal } & \multirow{2}{*}{\multicolumn{2}{|c|}{$\begin{array}{c}\log _{10} I D_{50} / g \\
\text { brain }\end{array}$}} & \multicolumn{2}{|c|}{$\log _{10}$ removal } & \multirow{2}{*}{\multicolumn{2}{|c|}{$\begin{array}{c}\log _{10} S_{50} / g \\
\text { brain }\end{array}$}} & \multicolumn{2}{|c|}{$\log _{10}$ removal } \\
\hline Sample & & Step & Total & & & Step & Total & & & Step & Total \\
\hline BSE spike & 8.0 & NA & NA & 6. & & NA & NA & & $0 \pm 0.2$ & NA & NA \\
\hline $\mathrm{NaOH}$ treatment & 6.3 & 1.7 & 1.7 & 5. & & 1.5 & 1.5 & & $6 \pm 0.3$ & 2.4 & 2.4 \\
\hline DE filtration & $\leq 5.2$ & $\geq 1.1$ & $\geq 2.8$ & $\leq 4$ & & $\geq 1.4$ & $\geq 2.9$ & & $3 \pm 0.1$ & 1.3 & 3.7 \\
\hline $\mathrm{H}_{2} \mathrm{O}_{2}$ bleaching & $\bar{N} \mathrm{~N}$ & $\bar{N} A$ & $\overline{N A}$ & $\leq 3$ & & $\geq 0.6$ & $\geq 3.5$ & & $0 \pm 0.4$ & 0.3 & 4.0 \\
\hline Final product & ND & NA & NA & $\leq 2$ & & $\geq 0.8$ & $\geq 4.3$ & & $0 \pm 0.6$ & 0.0 & 4.0 \\
\hline
\end{tabular}


$\log _{10}$ of BSE infectivity. To increase sensitivity of the mouse bioassay, we removed heparin by centrifuging samples $\left(20,000 \times g, 1 \mathrm{hr}, 4^{\circ} \mathrm{C}\right)$, washed the pellets, resuspended them in inoculation buffer, and inoculated mice as described. We tested brains of all mice for $\mathrm{PrP}^{\mathrm{TSE}}$ as reported previously (5). We detected residual infectivity in all aliquots, including the final product. $\mathrm{NaOH}$ treatment removed $1.5 \log _{10}$ of BSE infectivity. We estimated removals by other steps. DE filtration removed $\geq 1.4 \log _{10}$ of BSE infectivity. The hydrogen peroxide bleaching and methanol precipitation (final product) steps each removed $<1 \log _{10}$ of infectivity, considered negligible. Thus, cumulatively, scaled-down heparin purification removed a total of $\geq 2.9 \log _{10}$ of BSE infectivity; $\mathrm{NaOH}$ treatment and DE filtration were the only effective steps.

We also quantified residual $\mathrm{PrP}^{\mathrm{TSE}}$ in each sample using the real-time quaking-induced conversion (RTQuIC) assay with hamster-sheep chimeric prion protein (9) as substrate, expressing results as $\log _{10} 50 \%$ seeding doses $\left(\mathrm{SD}_{50}\right)$, as reported previously (5). We detected $\mathrm{PrP}^{\mathrm{TSE}}$ in unspun BSE spike and $\mathrm{NaOH}$-treated samples but only inconsistent signals in aliquots from successive steps (data not shown). To increase sensitivity and remove heparin interfering with RT-QuIC at low concentrations of PrP ${ }^{\mathrm{TSE}}$, we centrifuged all samples as we did previously. To quantify PrPTS, we resuspended pellets and serially diluted each sample in phosphate-buffered saline $0.05 \%$ sodium dodecyl sulfate, adding $2 \mu \mathrm{L}$ of each dilution to seed RT-QuIC, each dilution into quadruplicate wells (see $\log _{10} \mathrm{SD}_{50}$ values in Table). $\mathrm{NaOH}$ treatment removed $2.4 \log _{10}$ of PrPTE and DE filtration steps removed $1.3 \log _{10}$ of PrP $P^{\mathrm{TSE}}$. Hydrogen peroxide bleaching and methanol precipitation reduced $\mathrm{PrP}^{\mathrm{TSE}}$ by only negligible amounts. Thus, processing from crude heparin to final pharmaceutical heparin cumulatively removed $3.7 \log _{10}$ of spiked PrPTSE.

We showed previously, using a rodent-adapted scrapie agent, that heparin processing removed 3.6 $\log _{10}$ of scrapie infectivity and $3.4 \log _{10}$ of PrPTSE (5). Here, we report studies with the more relevant BSE agent itself, showing similar reduction by $3.7 \log _{10}$ of PrPTSE. We could demonstrate only $\geq 2.9 \log _{10}$ reduction in infectivity, because the starting titer of the BSE-infected brain homogenate was low. However, we detected both residual BSE infectivity and PrP ${ }^{\mathrm{TSE}}$ seeding activity after final steps of processing, so our model process did not yield sterile heparin. We found $\mathrm{NaOH}$ treatment and DE filtration to be the most effective steps for removing both BSE infectivity and $\mathrm{PrP}^{\mathrm{TSE}}$ seeding activity, consistent with previous results using scrapie agent.
Overall, our data suggest that typical heparin manufacturing is likely to remove substantial amounts of BSE agent. Furthermore, a probabilistic model assessing the VCJD risk for bovine heparin sourced from cattle in the United States and Canada estimated the risk to be very low (10). The demonstrated ability of a typical heparin purification process to remove substantial amounts of contaminating BSE agent, taken together with careful selection of low-risk bovine material to manufacture heparin, provides additional assurance of safety, supporting eventual reintroduction of bovine heparin to the US market.

\section{Acknowledgments}

We thank the staff in the Food and Drug Administration (FDA) Division of Veterinary Services for the outstanding care of animals. We owe special thanks to the staff in the laboratory of Byron Caughey at Rocky Mountain Laboratory, National Institute of Allergy and Infectious Diseases, National Institutes of Health, Hamilton, Montana, for giving us the recombinant constructs.

The FDA Animal Care and Use Committee approved all animal studies (ASP \#2015-07). Intramural grants from the FDA supported the work.

\section{About the Author}

Dr. Bett is a chemist at the US Food and Drug Administration, Silver Spring, Maryland, USA. His research focuses on protecting biological products from contamination with TSE agents to advance public health.

\section{References}

1. Will RG, Ironside JW, Zeidler M, Estibeiro K, Cousens SN, Smith PG, et al. A new variant of Creutzfeldt-Jakob disease in the UK. Lancet. 1996;347:921-5. https:/ / doi.org/10.1016/ s0140-6736(96)91412-9

2. US Food and Drug Administration. FDA encourages reintroduction of bovine-sourced heparin [cited 2019 Dec 15]. https://www.fda.gov/Drugs/DevelopmentApprovalProcess/Manufacturing/ucm600976.htm

3. Keire D, Mulloy B, Chase C, Al-Hakim A, Cairatti D, Gray E, et al. Diversifying the global heparin supply chain: reintroduction of bovine heparin in the United States? Biopharm Int. 2015;28:36-42 [cited 2019 Dec 13]. http:/ / www.pharmtech.com/diversifying-global-heparinsupply-chain-reintroduction-bovine-heparin-united-states

4. World Organization for Animal Health. BSE situation in the world and annual incidence rate (1989-31/12/2016) [cited 2019 Dec 18]. https:/ / www.oie.int/en/animal-health-in-theworld/bse-situation-in-the-world-and-annual-incidence-rate

5. Bett C, Grgac K, Long D, Karfunkle M, Keire DA, Asher DM, et al. A heparin purification process removes spiked transmissible spongiform encephalopathy agent. AAPS J. 2017;19:765-71. https:// doi.org/10.1208/s12248-017-0047-y

6. Castilla J, Gutiérrez Adán A, Brun A, Pintado B, Ramírez MA, Parra B, et al. Early detection of PrPres in BSE-infected 
bovine PrP transgenic mice. Arch Virol. 2003;148:677-91. https:/ / doi.org/10.1007/s00705-002-0958-4

7. Bett C, Piccardo P, Cervenak J, Torres J-M, Asher DM, Gregori L. Both murine host and inoculum modulate expression of experimental variant Creutzfeldt-Jakob disease. J Gen Virol. 2018;99:422-33. https:/ / doi.org/ 10.1099/jgv.0.001017

8. Gray JG, Dudas S, Czub S. A study on the analytical sensitivity of 6 BSE tests used by the Canadian BSE reference laboratory. PloS One. 2011;6:e17633. https://doi.org/10.1371/ journal.pone.0017633

9. Orrú CD, Groveman BR, Raymond LD, Hughson AG, Nonno R,Zou W, et al. Bank vole prion protein as an apparently universal substrate for RT-QuIC-based detection and discrimination of prion strains. PLoS Pathog. 2015;11:e1004983. https://doi.org/10.1371/journal.ppat.1004983

10. Huang Y, Forshee RA, Keire D, Lee S, Gregori L, Asher DM, et al. Assessment of risk of variant Creutzfeldt-Jakob disease (vCJD) from use of bovine heparin. Pharmacoepidemiol Drug Saf. 2020;29:575-81. https://doi.org/10.1002/pds.4982

Address for correspondence: Cyrus Bett, US Food and Drug Administration, 10903 New Hampshire Ave, Silver Spring, MD 20993, USA; email: cyrus.bett@fda.hhs.gov

\section{Undetected Circulation of African Swine Fever in Wild Boar, Asia}

\section{Timothée Vergne, Claire Guinat, Dirk U. Pfeiffer}

Author affiliations: National Veterinary School of Toulouse, Toulouse, France (T. Vergne, C. Guinat); Institut National de Recherche pour l'Agriculture, l'Alimentation et l'Environnement, Toulouse (T. Vergne, C. Guinat); City University of Hong Kong, Hong Kong, China (D.U. Pfeiffer)

\section{DOI: https://doi.org/10.3201/eid2610.200608}

African swine fever is a growing threat to the livestock industry. We examined data indicating that in most countries in Asia, most notified events were related to farm outbreaks; meanwhile, only a few wild boar cases were reported. We hypothesize the virus circulates unnoticed in wild boar populations in Asia.

A frican swine fever (ASF) is one of the greatest threats to the livestock industry worldwide. Since 2007, ASF virus (ASFV) has been reported in 34 countries in Europe and Asia (1). Some strains of ASF can be associated with case-fatality ratios of almost $100 \%$ and with economic damage caused by trade disruptions (2). The absence of a safe and effective vaccine and the evolving understanding of the epidemiologic role of wild boar have complicated efforts to control this disease (3).

Observations from the Russian Federation, Ukraine, and Romania have suggested that ASFV primarily circulates among small pig farms and spills over into commercial farms occasionally and into wild boar populations regularly (4). However, more recent reports from several countries in Europe, including the Baltic states and Belgium, suggest the virus might maintain itself within wild boar populations and occasionally spill over into domestic pig farms. This newly described epidemiologic cycle proposes direct transmission among wild boar and indirect intraspecific transmission through contaminated wild boar carcasses (4). This cycle also suggests the persistence of ASFV in wild boar populations even in the context of low wild boar density and despite high death rates caused by the disease (5).

In August 2018, ASFV was detected in China, the leading pig producer worldwide. Since then, the virus has also been reported in Mongolia, Vietnam, Cambodia, Hong Kong, North Korea, South Korea, Laos, Myanmar, the Philippines, Timor-Leste, Papua New Guinea, Indonesia, and India (1). The large-scale spread of ASFV demonstrates the challenges of controlling the disease in this region, which has a high density of domestic pigs, a high proportion of low biosecurity farms, a widespread practice of feeding pigs with food waste (6), and a complex pork trade network involving wet markets and slaughterhouses with poor hygiene (2).

Maps of predicted habitat suitability suggest that most areas of East and Southeast Asia are highly suitable for wild boar (7). Although information is limited about the spatial distribution of wild boar in Asia, studies suggest that in some regions of China, wild boar density could be similar (8) to that of eastern Poland, where ASFV has circulated in wild boar for $\geq 5$ years (9). Reports of crop losses caused by wild boar in China (10) indicate that close interactions between wild boar and human activities occur in the region.

The widespread presence of ASFV in pigs in Asia implies regular environmental spillover from the pig supply chain. Therefore, it is highly likely that ASFV is already widely circulating within some wild boar populations in Asia, causing substantial wild boar death. Because life expectancy of ASFV-infected 